\title{
Optical polarimetry and photometry of young sun-like star LO Peg
}

\author{
J. C. Pandey ${ }^{1}$, B. J. Medhi ${ }^{1}$, and R. Sagar ${ }^{1}$ \\ ${ }^{1}$ Aryabhatta Research Institute of Observational Sciences, Nainital, India -263129 \\ email: jeewan@aries.res.in
}

\begin{abstract}
We have carried out the B,V and R-band polarimetric and V-band photometric study of the star LO Peg. Our analysis reveal that LO Peg is highly polarized among the sunlike stars. The degree of polarization and polarization position angle are found to be rotationally modulated. The levels of polarization observed in LO Peg could be the result of scattering of an anisotropic stellar radiation field by an optically thin circumstellar envelope or scattering of the stellar radiation by prominence-like structures. The long term photometric observations of LO Peg indicate three independent groups of spots are present on the surface of LO Peg.
\end{abstract}

Keywords. Stars: activity, stars: late-type, stars: spots, techniques: photometric, techniques: polarimetric

\section{Introduction}

LO Pegasi (LO Peg) is a single, young, K3V-K7V-type and a member of the Local Association (Montes et al. 2001; Pandey et al. 2005). It is one of the fast-rotating active stars with a period of 0.42 days. LO Peg shows strong $\mathrm{H} \alpha$ and $\mathrm{Ca}$ II $\mathrm{H}$ and $\mathrm{K}$ emission lines (Jeffries et al. 1994). Evidence of an intense downflow of material and optical flaring on LO Peg had presented by Eibe et al. (1999). Zuckerman et al. (2004) have identified LO Peg as a member of a group of 50-Myr-old stars that partially surround the Sun. In this contribution, we have investigated the polarimetric and long term photometric observations of LO Peg.

\section{Observations and data reductions}

The broad-band $\mathrm{B}, \mathrm{V}$ and $\mathrm{R}$ polarimetric observations of $\mathrm{LO}$ Peg have been made in between 2007 October 19 and December 19 using ARIES Imaging Polarimeter (Rautela et al. 2004), mounted on the Cassegrain focus of the 104-cm Sampurnanand telescope (ST) of Aryabhatta Research Institute of Observational Sciences, Nainital. Details of polarimetric data reduction are given in Medhi et al. 2010 and Pandey et al. 2009. For calibration of polarization angle zero-point, we observed highly polarized standard stars. For instrumental polarizations, we observed standard unpolarized stars. Photometric observations in V-band were also taken from ST using $2 \mathrm{k} \times 2 \mathrm{k}$ CCD (see Pandey et al. 2005) during year 2001 and 2002. We have also used the All Sky Automated Survey (ASAS; Pojmanski (2002)) data for our study. We have used only 'A' and 'B' graded data. The ASAS survey has longer span of V-band photometry of 6.5 years. The total time span of the observations is 8 years, which is useful to observe any long term variations as well as migration of spots on the stellar surface.

\section{Polarization}

We have fold the polarimetric data using the ephemeris HJD $=2448869.93+0.42375$ E. Top and middle panels of Figure 1 show the variations of degree of polarization 


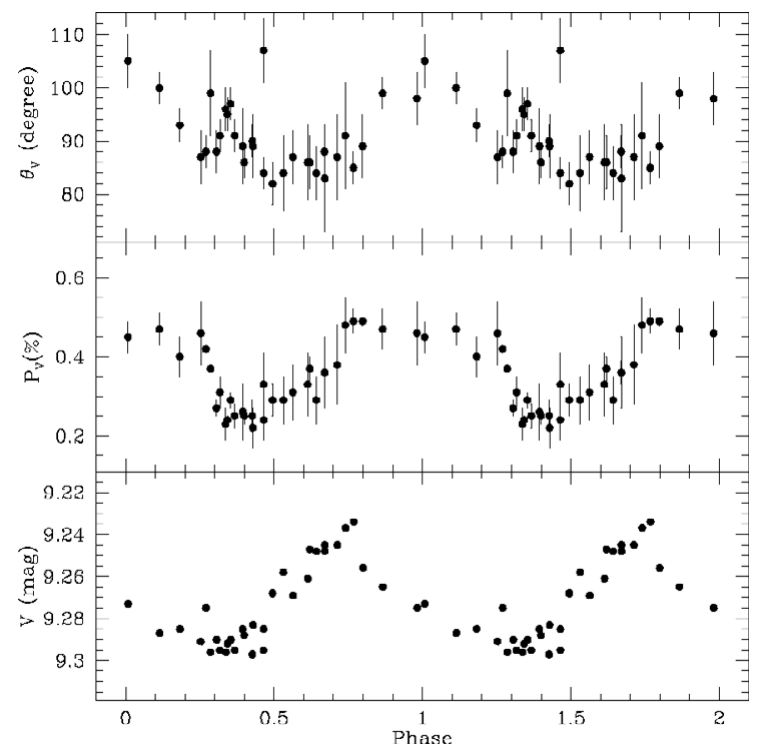

Figure 1. Bottom Panel: V-band light variation, Middle panel: V-band polarization variation and Top panel: Position angle $\left(\theta_{V}\right)$ variation with rotational phase of the star LO Peg. The data are folded with rotational period of $0.42 \mathrm{~d}$

$\left(\mathrm{P}_{V}\right)$ and position angle $\left(\theta_{V}\right)$ in V-band. Both are found to be rotationally modulated. The average values of degree of polarization in $\mathrm{B}, \mathrm{V}$ and $\mathrm{R}$ bands are found to be $0.387 \pm 0.004 \%, 0.351 \pm 0.004 \%$ and $0.335 \pm 0.003 \%$, respectively. However, the average values of polarization position angle are found to be $88^{\circ} \pm 1^{\circ}, 91^{\circ} \pm 1^{\circ}$ and $91^{\circ} \pm 1^{\circ}$ in $\mathrm{B}$, $\mathrm{V}$ and $\mathrm{R}$ band, respectively. The nearly similar values of polarization position angle in each band indicates that the scattering geometry is identical at all wavelengths. Below, we investigate the origin of polarization in LO Peg.

LO Peg is located at a distance of $25 \mathrm{pc}$, and therefore has a negligible reddening. It is quite natural to assume that the observed polarization in LO Peg is not foreground in origin. Further, the observed polarization in any distant star located near the Galactic plane may have small negligible interstellar component. Further, the existence of a timedependent polarization is a well-established criterion for intrinsic polarization. Huovelin \& Saar 1991 showed that the B, V and R-band polarization due magnetic filed should be less than $0.16 \%$ for a K-type dwarf having $2.7 \mathrm{k}$ Gauss magnetic filed and total spot area of $\approx 24 \%$. The observed values of polarization in LO Peg are more than $0.16 \%$. Therefore, the polarization in LO Peg could not be magnetic in origin. The observed polarization in LO Peg was found to decrease towards longer wavelength. This could be due to either selective absorption by circumstellar dust, which grows towards shorter wavelengths or wavelength-dependent albedo, which decreases towards longer wavelengths resulting less scattering and thus polarization. We have also investigated that whether the polarization in LO Peg due to cool prominence like structures which are located at the co-rotation radius $\left(\mathrm{r}_{c}\right)$. Pfeiffer $(1978)$ showed that polarization due prominence like structure is $\mathrm{P}=$ $\mathrm{N} \sigma \mathrm{f} /\left(\mathrm{r}_{c}^{2}+\mathrm{N} \sigma \mathrm{f}\right)$. Here, $\mathrm{N}$ is number of scatterer, $\mathrm{f}$ is angular scattering function and $\sigma$ is scattering cross-section. Using above relation, the amount of scatterers are determined to be $\sim 10^{48}$. If the cloud contains mostly hydrogen, this amounts to about $\sim 10^{24} \mathrm{~g}$, which is only an order larger than the mass-loss rate $\left(2.0 \times 10^{-11} \mathrm{M}_{\odot} \mathrm{yr}^{-1}\right)$.

Variation of $\mathrm{V}$-band magnitude is shown in bottom panel of Fig. 1. Rotational modulation is clearly visible. It appears that the variation in V-band light is correlated with the 

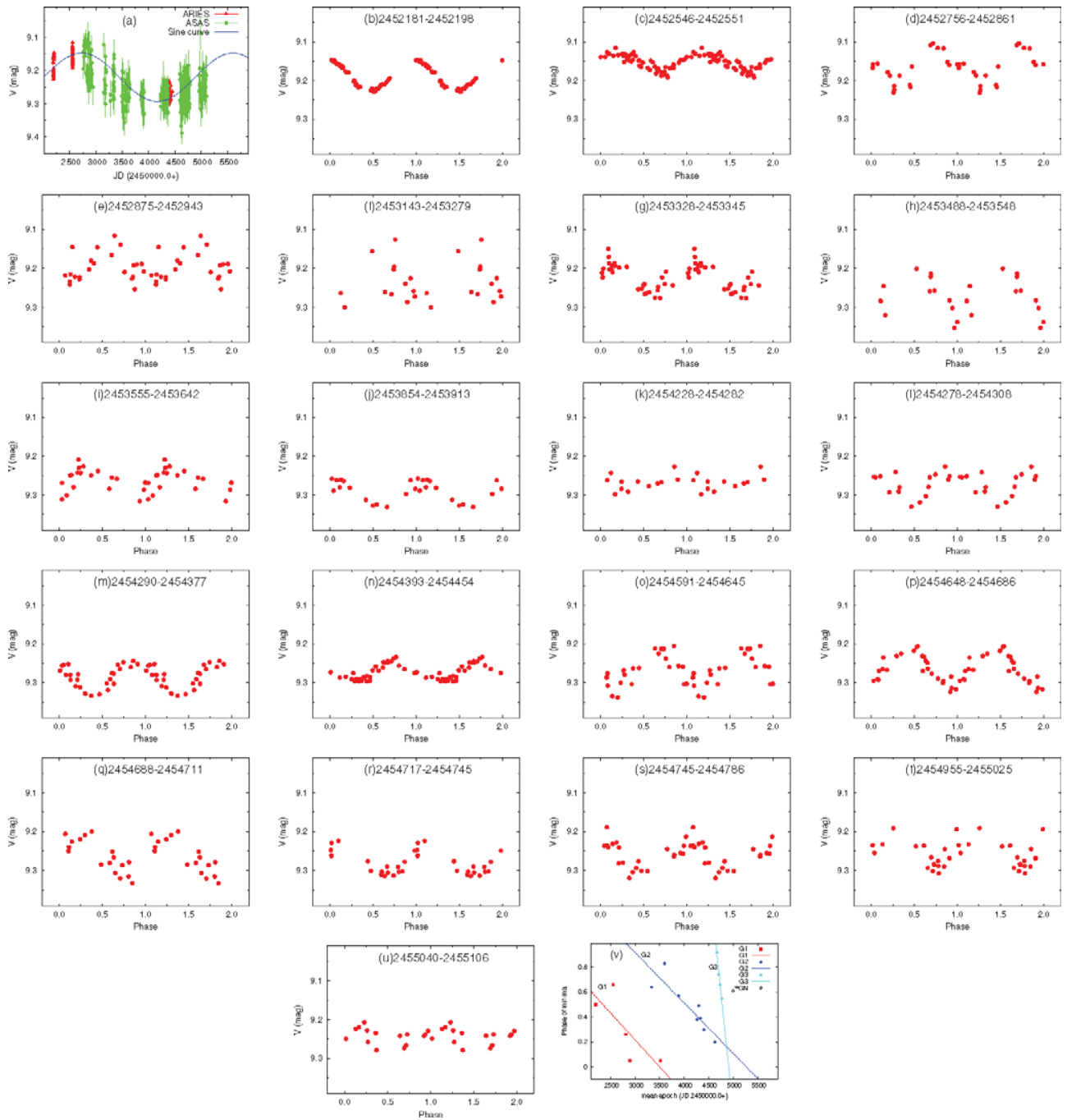

Figure 2. The top-first panel is V-band light curve as function of JD. From top-second to bottom-third panels are light curves as a function of rotational phase at 20 different epochs in chronological order. The epochs are mentioned at top of each diagram. The bottom forth panel shows a plot between minima of phase and mean epoch of observations.

V-band polarization. The significance of correlation has been calculated by determining the linear correlation coefficient $r$ between the magnitude and the degree of polarization. The values of $\mathrm{r}$ between $\mathrm{V}$-band magnitude and $\mathrm{P}_{V}$, and $\mathrm{V}$-band magnitude and $\theta_{V}$ are found to be 0.47 and 0.45 with the corresponding probability of no correlation being 0.008 and 0.013 , respectively. Star was brighter when $\mathrm{P}_{V}$ was higher. This implies that the surface brightness inhomogeneity seems to play a major role in the polarization variability of LO Peg. Inhomogeneities in the distribution of both the material in the circumstellar envelope and brightness on the surface of the illuminating star can provide the necessary asymmetry, and hence produces a net polarization in the integrated light. The mechanism which can produce periodic linear polarization variability is Rayleigh or Thomson scattering in a non-spherically symmetric distribution of circumstellar material. Recently, Al-Malki et al. (1999) showed that a $\sim 0.5 \%$ of polarization can be achieved from an anisotropic light source scattered by a Maclaurian spheroidal envelope 
$\left(\mathrm{a}=\mathrm{b}=1\right.$ and $\mathrm{c} \approx 0.6$ ) with an optical depth of 0.1 and an angle of inclination of $60^{\circ}$, which is similar to that observed in LO Peg.

\section{Long term photometric variability}

V-band light curve of the star LO Peg is shown in Fig. 2(a). Long variations are clearly seen. The continuous line is least -squared fit sine curve in the form of $A \sin (2 \pi f x+\phi)$, where $A$ is semi-amplitude and $f$ is frequency of the wave. From sine curve fitting, the period of long variation is found to be $\sim 8$ years which may be similar to the 11-years solar-cycle. Further, the entire $\sim 8$ years V-band data of LO Peg are divided into 20 epochs and folded with rotational period of $0.42 \mathrm{~d}$. The folded light curves at 20 different epochs are shown in Figs. 2(b) to 2(u). When comparing the light curves of LO Peg from one epoch to another, a shift in the phase of the minimum and a variable amplitude are quite evident. This could be due to the presence of dark spots on the surface of the star. The variable depth of minima could be due to the variable size of the of group of spots on the surface of LO Peg. We have determined the phase of minima $\left(\theta_{\text {min }}\right)$ of each light curve by least square fitting of the second order polynomial during the minimum phase. The plot between $\theta_{\min }$ and mean epoch of the observations is shown in Fig. 2 (v). The phases of the light minima directly indicate the mean longitude of the dominant groups of the spots. The presence of three groups of spots (say G1, G2 and G3) is clearly established by three well-separated straight lines. It appears that one more group is emerging on the surface of LO Peg (see open circle in Fig. 2(v), marked by GN) . We fit the relation $\theta_{\min }(t)=\omega t+\theta_{0}$ to each G1, G2 and G3 groups of spots separately to determine the angular velocity of $\operatorname{spot}\left(\right.$ in $\operatorname{deg}$ day $\left.^{-1}\right)$ or rate of phase shift $(\omega=2 \pi / P$, $P$ is migration period in days). Here, $\theta_{0}$ is reference spot longitude. The continuous lines in Fig. 2 (v) are least square fits of this above relation. Applying the above relation, the rate of phase shift for spots G1, G2 and G3 is determined to be $0.16^{\circ}$ day $^{-1}, 0.15^{\circ}$ day $^{-1}$ and $1.37^{\circ} \mathrm{day}^{-1}$, respectively. The corresponding migration period for spots G1, G2 and G3 is found to be $6.2,6.6$ and 0.7 yrs, respectively. The similar migration period of spots G1 and G2 indicates that spots are located at different longitudes rotate with almost the same angular velocity. The faster rotation of spot G3 could be due to its different latitudinal position.

\section{Conclusions}

We conclude that the high values of polarization observed in LO Peg require either a spheroidal envelope with an optical depth of 0.1 or a clumpy material (e.g. solar prominence-like structures) of mass of the order of $\sim 10^{10} \mathrm{M}_{\odot}$. From long term photometric data, we established that three groups of spots are present on the surface of LO Peg, out of which one spot is migrating faster than other two.

Acknowledgments. Many thanks to IAU for financial support to attend the symposium and ASAS for the V-band data.

\section{References}

Al-Malki M. B., Simmons J. F. L., Ignace R., Brown J. C., \& Clarke D., 1999, Astron. Astrophys, 347,919

Eibe M. T., Byrne P. B., Jeffries R. D., \& Gunn A. G., 1999, Astron. Astrophys, 341, 527

Huovelin J., \& Saar S. H., 1991, Astrophys. J., 374, 319

Jeffries R. D. et al. 1994, Mon. Not. Roy. Astron. Soc., 270, 153 
Medhi B. J., Maheswar G., Pandey J. C., \& Sagar R., 2010, Mon. Not. Roy. Astron. Soc., 403, 1577

Montes D., et al. 2001, Mon. Not. Roy. Astron. Soc., 328, 45

Pandey J. C., Singh K. P., Drake, S. A., \& Sagar R. 2005, Astron. J., 30, 490

Pandey J. C., Medhi B. J., Sagar R., \& Pandey A. K. 2009, Mon. Not. Roy. Astron. Soc., 30, 490

Pfeiffer R. J., 1979, Astrophys. J., 232, 181

Pojmanski G. 2002, Acta Astronomica, 52,397

Rautela B. S., Joshi G. C., \& Pandey J. C., 2004, Bull. Astron. Soc. India, 32, 159

Zuckerman B., Song I., \& Bessell M. S., 2004, Astrophys. J., 613, L65 